\title{
Maturidade mental em gêmeos univitelinos
}

\author{
Mental maturity in monozygotic twins
}

\section{Rachel Schlindwein-Zanini ${ }^{1}$}

\begin{abstract}
RESUMO
Introdução. Os gêmeos geralmente despertam o encantamentoe acuriosidade.Oestudodosgêmeosedassuasfamíliasforneceuma ferramenta para entender as participações genéticas eambientais na manifestação de suas características. Esta pesquisa visa avaliar a maturidademental em gêmeos monozigóticos, como contribuição para as decisões de profissionais de saúde, pais e educadores. Método.Duas crianças gêmeas monozigóticas, do sexo masculino com três anos e 10 meses de idade, freqüentadoras da pré-escola. $\mathrm{O}$ instrumento aplicado foi a Escala de Maturidade Mental Colúmbia (CMMS). Também foi realizada entrevista psicológica com a mãe dos gêmeos. Resultados. Os gêmeos obtiveram o mesmo índice de maturidade de $5 \mathrm{I}$, entre 5 anos a 5 anos e 5 meses, sendo um bom resultado em termos de maturidade mental e raciocínio geral. Conclusão. Ambos os gêmeos mostraram o mesmo índice dematuridadede5l,oquesignificabom desempenhonastarefase sinalizando compatibilidade com a idade cronológica deles.
\end{abstract}

Unitermos: Maturidade. Gêmeos. Psicologia. Neuropsicologia.

Citação: Schlindwein-Zanini R. Maturidade mental em gêmeos univitelinos.

\section{SUMMARY}

Introduction. The twins generally bewitch and awake the curiosity. The study on twins and his families provides a tool to understand thegeneticandenvironmentalcontributionstohis characteristics and behavior. This research aims to evaluate the mental maturity in monozygotic twins and to contribute with the professionals of health, parents and educators in their decisions. Method. Two monozygotic twins, males, 3 years and 10 months old, in preschool. The applied instrument was Columbia Mental Maturity Scale (CMMS). Also psychological interview with the mother was carried out. Results. The twins achieved $5 \mathrm{I}$ in the index of maturity, being inter 5 years the 5 years and 5 months, meaning a good resulted in terms of mental maturity and general reasoning. Conclusion. Both twins showed the same index of maturity 5 I that means a good performance and suggests a compatibility with their chronological age.

Keywords: Maturity. $\quad$ Twins.
Neuropsychology.

Citation: Schlindwein-Zanini R. Mental maturity in monozygotic twins.
Trabalho realizado na Pontifícia Universidade Católica do Rio Grande do Sul - PUCRS.

1. Neuropsicóloga, Doutora em Ciências da Saúde pela Faculdade de Medicina (FAMED) da Pontifícia Universidade Católica do Rio Grande do Sul - PUCRS. Pós-Doutorado em Psicologia pela Universidade Federal de Santa Catarina - UFSC.
Endereço para correspondência: Rachel Schlindwein-Zanini R. Lauro Linhares 2123, torre 1, sala 612 88036-002 Florianópolis, SC E-mail: rachelsz@floripa.com.br 


\section{INTRODUÇÃO}

Osgêmeosgeralmentedespertamoencantamento e a curiosidade das pessoas, sendo que cada região, cultura e religião tem suas teorias sobre os gêmeos.

A partir do nascimento, os gêmeos chamam a atenção dos pais, de amigos e parentes. Famílias se encantameinconscientementepodemtransformaras vidas de seus filhos gêmeos em uma só. Este comportamentopodedenunciaradificuldadedevê-loscomo pessoasdistintas, apesardapreocupaçãodospaiscom a individualidade destas crianças. Gêmeos idênticos porvezes recebem nomes e roupas semelhantes, freqüentam o mesmo ambiente social e escolar è̀s vezes ainda são confundido um pelo outro. Entretanto, percebem o mundo de maneira singular e única. $A$ proximidadeeasemelhançafísica podeminfluenciar, inclusive prejudicialmente, a formação da identidade das crianças'. Há a constatação de que gêmeos idênticos são tratados de modo mais semelhante do que osfraternos, inclusivequandocriadosseparadamente porpais diferentes relatam histórias parecidas de criação ${ }^{2}$. Assim, as diferenças entre gêmeos idênticos são atribuídas a efeitos ambientais ${ }^{3}$.

Osgêmeospodemserbivitelinos(dizigóticos), ou podem sergêmeos univitelinos (monozigóticos). Irmãos idênticos são os que compartilharam o mesmo útero, frutos da fertilização de um único óvulo porumespermatozóide,ocorrendoumafissãodozigoto formado. Uma fissão dá origem a dois gêmeos idênticos, trêsfissõesatrigêmeos, quatroaquadrigêmeos e assim sucessivamente 4 .

Apesar de os gêmeos idênticos monocoriônicosgeralmentenasceremcompesos diferentesentre si, são os que mais tarde vão apresentar maiores semelhanças psicológicas 5 .

O estudo dos gêmeos e de suas famílias fornece uma ferramenta altamente útil para entender as origens genéticas e ambientais dos traços que apresentam, fornecendo um recurso valioso para compreenderascomplexidadesdodesenvolvimento psicológico infantil6.

Tendo em vista a importância do tema e a carênciadepesquisasdestinadasaaspectoscognitivosem gemelares,esteestudovisaavaliaramaturidademental em gêmeos univitelinos, com o intuito de subsidiar os atos dos profissionais de saúde, pais e educadores.

\section{Aspectos psicológicos e neuropsicológicos dos gêmeos}

A neuropsicologia é uma ciência que abrange a cognição, o comportamento e a atividade do
sistemanervoso.Aavaliaçãoneuropsicológicainfantil, diferenciada da do adulto, possui características próprias, tendo em vista a evolução contínua, inerenteao desenvolvimentofísico, cognitivo, cerebral, linguísticoepsico-social.Nessesentido,umapesquisa que mostra os resultados de uma avaliação neuropsicológica baseada nos conceitos fundamentais propostos por Luria, em três grupos gemelares, de oitoadezanosdeidade, pareadosemseusdesempenhosmotores, perceptuais, cognitivosdelinguagem, memória, habilidade numérica e leitura/escrita ${ }^{7}$. Os resultadosindicamqueasdiferençasdedesempenho entre os pares de gêmeos incidem mais sobre os aspectospsico-sociaisdoquepropriamentesobreaquisição. O domínio do código lingüístico, a elaboração de conteúdos simbólicos e a motivação foram resultados diferenciadosnesteestudocomparativo,onde sedestacoutambémaimportânciadomeioambientenoprocessodedesenvolvimentodasfunçõescorticais e na aquisição de aprendizagem escolar.

Otrabalho dePavan pesquisou a relaçãoentre a inteligência eo patrimônio hereditário, comparandodoisgruposdegêmeos:univitelinosebivitelinos ${ }^{8}$. O grupo sujeito tinha 10 pares de gêmeos idênticos e o controle, 13 de fraternos. Os indivíduos eram do sexo masculino, com idades entre 6 a 11 anos, e todos os pares foram criados juntos. Observou-se que há uma maior harmonia entre os pares univitelinos do que entre os fraternos. Os univitelinos parecem ter facilidade para criar intimamente situações comuns enquanto que os segundos procuram quase semprecriar situações diferentes. A todos os sujeitos da pesquisa foi aplicado o teste de Raven, série para crianças. Concluiu-se haver uma relação direta entre a inteligência do ponto de vista qualitativo e patrimônio hereditário ${ }^{8}$.

Exceto as dificuldades no desenvolvimento relacionadas à prematuridade, gêmeos e trigêmeos e outros múltiplos têm a mesma capacidade intelectual que as demais crianças. Visto que tantos múltiplos nascem prematuros, podem apresentar, durante os primeiros anos de vida, os atrasos associados à prematuridade ou ao baixo peso ao nascer. Mesmo assim, a maioria supera os atrasos na idadeescolar.Masmúltiplos desenvolvemashabilidades intelectuais e de linguagem em um contexto diferente daquele vivenciado por outras crianças. Crescem ao lado de um ou mais irmãos da mesma idade, e, portanto, geralmente têm menos interação individual com adultos, o que gera vantagense desvantagens ${ }^{9}$. 
Ser criado junto pode significar aspectos distintos para cada par de gêmeos. Há indícios de que gêmeos monozigóticos criados juntos tenham um ambientemaissemelhantequeosdizigóticoscriados juntos, pois tendem a estudar mais na mesma classe e a partilhar mais atividades extracurriculares ${ }^{5}$. A condição de gêmeos lhes dá vantagem de ter um parceiro com o qual praticar alternadamente os papéisdelíder-seguidoreaartedorevezamento,queé essencial para o desenvolvimento da conversação?

Aos 30 meses de idade, os meninos gêmeos podemestar8mesesemdesvantagemnodesenvolvimentoemrelaçãoascriançasnão-gêmeas(singletons) e as meninas gêmeas na linguagem expressiva e 6 meses de desvantagem na compreensão verbal. Há também 5 mesesdedesvantagemnojogosimbólico, relacionada à linguagem. Alíngua"secreta"foicaracterística na maioria dosmeninosgêmeos, masnãoas meninas.Nessesentido,foramexaminadosmeninos gêmeosentre38-53meseseossingletons, utilizando a Escala de Maturidade Mental Colúmbia, no nível de início do ensino pré-escolar. Os meninos gêmeos tiveram mais dificuldades na articulação e na sociabilidade, mas após 8 meses no ensino pré-escolar as crianças tiveram melhoras na sociabilidade ${ }^{10}$.

Antes da fala, gêmeos desenvolvem habilidades de comunicação não verbal altamente sofisticadas ${ }^{9}$. A imitação,ovocabulário,olúdicoeocomportamentosocialcominsightforampesquisadosem paresdegêmeos de ambos os sexos, com dois anos de idade, no Reino Unido. As diferenças individuais na habilidade imitativa eramdevidas,emparte,àherdabilidade(30\%),osfatores ambientaiscompartilhadosentregêmeos(42\%)eúnico a cada gêmeo (28\%). Os pais que incentivam a imitação podem, também, promover o desenvolvimento da linguagemedocomportamentosocialcominsight ${ }^{11}$.Foi identificado um comportamento verbalem gemelares, que chamaram de "efeito dos gêmeos", relacionado ao conceitoqueascriançastêmdesiprópriascomoequipe. Essa linguagem é composta de adaptações do idiomapadrão,usadasparaexpressararealidadeespecialdeles, incluindo termos como o "eu e eu também", "a gente" (opostode"vocês")eseusnomesprópriosunidosemseqüência, seguidos deverbos nosingular'. Desdeoinício davida,gêmeosemúltiplosdeordemsuperiortêmuma redecomplexaderelacionamentosparanegociar.Oque eles mais requerem dos pais é a admiração pelas habilidades sociais (como o revezamento), paciência no seu desenvolvimentodalinguagem(suaproduçãodovocabulário) quandoestiverem desvantagem comparativamente às outras crianças ${ }^{9}$.
Outro aspecto relevante para as crianças, especialmenteosgêmeos,éaqualidadedevida.Assim, ressalta-se a importância da QV para o bem-estar geral do indivíduo ${ }^{12}$.

Nem os gêmeos univitelinos são idênticos. Então, se for considerado o princípio que clones representamindivíduosiguais, nãoexistem clones. Mesmo quandoseconsiderargêmeosunivitelinos, umaforma de reprodução assexuada nos mamíferos, com $99,9 \%$ das características iguais, ocorrem mutações ${ }^{13}$.

Os gêmeos parecem preencher todos os requisitos para atender ao raciocínio básico dos estudos de genéticadocomportamento.Acomparaçãodegêmeos idênticosversusgêmeosfraternos,criadosjuntosouem separado, parecepermitiraconcretização,emambientenatural,detodososcontrolesexperimentaisnecessáriosparaoestudodeefeitosdoambienteedagenética ${ }^{3}$. Nesse sentido, cita-se a pesquisa Estudo de Minnesota de Gêmeos Criados em Separado (Minnesota Study of Twins Reared Apart - MISTRA) ${ }^{3}$. Bouchard retomou a análise dos dados da aplicação de um teste vocacional: a medida de correlação dos gêmeos idênticos criados em separado é da ordem de 0,50 , enquanto a medida decorrelaçãodosgêmeos dizigóticostambémseparados é da ordem de 0,0714. Considerando-se os dizigóticoscomoumgrupodecontrole, poder-se-iadizerque a diferença entre os grupos, da ordem de 0,43, é determinadaporefeitogenético.Resultadosdestamesma ordem de magnitude são encontrados para traços de personalidade, como extroversão e medidas de psicopatologia, entre muitas outras ${ }^{3}$.

Há um grande interesse científico acerca da influência do ambiente e a da genética na formação doindivíduo.Nessesentido, aspesquisascomgêmeos são valiosas, onde percebe-se a importância da interação entre fatores hereditários e ambientais na determinação do desenvolvimento do indivíduo.

Na realidade, há influência de ambos os aspectos, quepodemgerardiferentes reaçõesemcada pessoa, marcando-a como ser único e singular.

O objetivo deste estudo foi pesquisar a maturidade mental em gêmeos univitelinos.

\section{MÉTODO}

\section{Relato de Caso}

Duascriançasgêmeasunivitelinas, dosexomasculino, com três anos e 10 meses de idade, freqüentadoras da pré-escola, aqui identificadas como sendo Ee I.Apresentepesquisafoidesenvolvidanomunicípiode Florianópolis, Estado de Santa Catarina, Brasil. 
A entrevista com a mãe forneceu informações importantes, comoidadegestacionalde 35 semanas e 5 dias ao nascer, dados do nascimento, das atividades de vida diária e dinâmica familiar, principalmente. Ambas as crianças apresentam desenvolvimento neuropsicomotor (DNPM) normal, assim como no âmbito comportamental, inclusive alternam papéis de liderança entre si. Também mostravam boa integração social e desempenho escolar. O quadro clínico e nutricional é bom, I tinha 16,5 kg e E tinha 17 kg. Ambos mediam 1,08 m. Residiam com o pai e a mãe, que têm instrução superior.

O instrumento aplicado foi a Escala de MaturidadeMentalColúmbia(ColumbiaMentalMaturity Scale - $\left(\mathrm{CMMS}^{15}\right)$. É um teste individual, validado no Brasil, de fácil aplicação, quefornece uma estimativa da capacidade de raciocínio geral de crianças. Avalia especialmentecapacidadesquesãoimportantespara osucessonaescola, principalmenteaquelasparadiscernir as relações entre os vários tipos de símbolos ${ }^{14}$.

\section{RESULTADOS}

Na Escala de Maturidade Mental Colúmbia, ambos os gêmeos obtiveram índice de maturidade 51 , estando entre 5 anos a 5 anos e 5 meses. Na prancha 32, E e I identificam o par de pintinhos como gêmeos e não como diferentes dos demais no grupo mostrado na lâmina.

\section{DISCUSSÃO}

Os gêmeos apresentados neste estudo mostram várias semelhanças, como a questão genética, comofreqüentaramesmasalanoensinopré-escolar, além de serem igualmente estimulados pela família, inclusive a mãe das crianças é psicóloga e empenhaseemestimulá-los noâmbitocognitivo, social,cultural, motor e afetivo. Sendo assim, podem-se esperar resultados semelhantes na Escala de Maturidade Mental Colúmbia.

Na Escala de Maturidade Mental Colúmbia, ambos os gêmeos mostraram o índice de maturidade de 5 I. Isto quer dizer que estão $97 \%$ melhor que seu grupo etário. Em termos de raciocínio geral, eles estão entre os $14 \%$ das crianças que têm RPI (Resultado Padrão de Idade) entre 116 e 132. Sinalizando bons resultados, considerando que a idade cronológica deles é de 3 anos e 10 meses. Segundo a escala, I e E estavam em uma posição privilegiada, já que estavam $97 \%$ melhor que seu grupo etário, entre os $14 \%$ das crianças que têm RPI entre 116 e 132, já quedesempenharamamaioriadastarefascomêxito e compreenderam os comandos em tempo compatível (30 minutos cada indivíduo). As reações frente à aplicação da escala foram diferentes nas crianças. Emalgunsmomentos, I, possivelmenteno intuito de responderrapidamente, apontavaaprimeiraresposta do lado direito. Em outros momentos E queria ver a lâmina seguinte. Em ambas situações, esta examinadora os instruía a voltar aos comandos do teste.

É interessante citar que na prancha 32, onde há pintinhos amarelos sozinhos e um par de iguais, E e I identificaram-nos como gêmeos e não como diferentes dos demais no grupo mostrado na lâmina. Para fins quantitativos, o CMMS não indica a pontuação, masqualitativamentesugere-seconsiderar. Tal aspecto evidenciou a importância do irmão paracadagemelar,semqueacondiçãodegêmeosos excluíssem de um grupo social. Tal comportamento pode-se relacionar com o"efeito de gêmeos", que os une verbalmente mais ainda.

O CMMS mostrou-se útil na investigação da maturidade mental de gemelares. A partir dos resultados do CMMS, poderiam ser realizados outros testesnointuitodeinvestigar,emmaiorespecificidade, as áreas e potencialidades em que estas crianças diferem uma da outra ou são semelhantes.

Aomesmotempoemqueosgêmeosunivitelinossãoindivíduossingulares,sãosemelhantesentre si; entretanto, diferenciando-se dos demais por um vínculo único com um grande amigo: o irmão.

Desse modo, apesar das várias similaridades, os gêmeos são indivíduos diferentes em sua forma de pensar, sonhar, agir e amar. E assim devem ser considerados: únicos.

\section{CONCLUSÕES}

A partir do presente trabalho, conclui-se que na Escala de Maturidade Mental Colúmbia, ambos os gêmeos mostraram o mesmo índice de maturidade de $5 \mathrm{I}$, estando entre 5 anos a 5 anos e 5 meses, tendo bom desempenho nas tarefas e sinalizando compatibilidade com a idade cronológica deles.

\section{AGRADECIMENTOS}

Ao Prof. Dr. Livre-Docente (USP) Antonio Pedro Schlindwein.

\section{REFERÊNCIAS BIBLIOGRÁFICAS}

1. Jabbour C, Marques L. Gêmeos: onde está a semelhança? Rio de Janeiro: Editora Papel e Virtual, 2000, 110 p.

2. Harris JR. The nurture assumption. New York: The Free Press, 1998,462 p. 
3. Bussab VSR. Fatores hereditários e ambientais no desenvolvimento: a adoção de uma perspectiva interacionista. Psicol Reflex Crit 2000;13(2):233-43.

4. Borém A, Vieira MLC. Glossário de Biotecnologia. Viçosa: Editora Folha de Viçosa, 2005, vol. 1, 177 p.

5. Spitz E. Des jumeaux bien dociles. La Recherche 1996;283:73-5.

6. van den Bree MB, Rice F, Fowler TA, Shelton KH, Lifford

KJ, Scourfield J, et al. The Cardiff Study of all Wales and North

West of England Twins (CaStANET): a longitudinal research program of child and adolescent development. Twin Res Hum Genet 2007;10(1):13-23.

7. Tabaquim MLM, Guimarães CA, Abramides DVM, Ciasca SM Avaliação neuropsicológica em grupos gemelares. Temas Desenvol 2001;10(56):5-11.

8. Pavan ML. Um estudo das diferenças de inteligência entre gêmeos idênticos e fraternos mediante o teste de Raven. J Bras Psicol 1964;1(2):77-90.

9. Malmstrom PM, Poland J. Criando filhos gêmeos. São Paulo: M Books, 2004, 298 p.
10. Hay DA, Prior M, Collett S, Williams M. Speech and language development in preschool twins. Acta Genet Med Gemellol (Roma) 1987;36(2):213-23.

11. McEwen F, Happé F, Bolton P, Rijsdijk F, Ronald A, Dworzynski K, et al. Origins of individual differences in imitation: links with language, pretend play, and socially insightful behavior in two-year-old twins. Child Dev 2007;78(2):474-92.

12. Schlindwein-Zanini R, Portuguez MW, Costa DI, Marroni S, da Costa JC. Epilepsia Refratária: Repercussões na Qualidade de Vida da Criança e de seu Cuidador. J Epilepsy Clin Neurophysiol 2007;13(4):159-62.

13. Pavan C. Entrevista concedida a Luciano Dias de Moraes. Informativo JR USP 1997;6:1-5.

14. Bouchard TJ Jr. Twin studies of behavior. In: Schimitt A, Atzwanger K, Grammer K, Schäfer K. New aspects of human ethology. New York: Plenum Press, 1997, 121-40.

15. Burgemeister BB, Blum LH, Lorge I. Padronização Brasileira da Escala de Maturidade Mental Colúmbia. In: Burgemeister BB, Blum LH, Lorge I. Escala de Maturidade Mental Colúmbia: Manual para Aplicação e Interpretação. São Paulo: Casa do Psicólogo, 2001, 68 p. 